\title{
Author Correction: Immune-orthogonal orthologues of AAV capsids and of Cas9 circumvent the immune response to the administration of gene therapy
}

Ana M. Moreno, Nathan Palmer (D), Fernando Alemán, Genghao Chen, Andrew Pla, Ning Jiang, Wei Leong Chew (D), Mansun Law and Prashant Mali iD

Correction to: Nature Biomedical Engineering https://doi.org/10.1038/s41551-019-0431-2, published online 22 July 2019.

In the version of this Article originally published, in the heading for Fig. 1g 'DNA-targeting CRISPR effectors (285)', '285' should have read '284'. In Fig. 3b,c,e and f, the final $x$ axis label, 'AAV5-SaCas9' in blue, for the first dose was incorrect; the correct label is 'AAV5-SpCas9'. These figures have now been corrected. In addition, the caption for Supplementary Fig. 2 stated that 89 Cas13a, b and c orthologs were used; the correct number is 84 . In Supplementary Table 1 the Cpf1 and Cas 13 sequences were missing. The Supplementary Information and Supplementary Table files have now been corrected. 